\title{
Postcricoid Region
}

National Cancer Institute

\section{Source}

National Cancer Institute. Postcricoid Region. NCI Thesaurus. Code C54221.

The portion of the hypopharynx that is located posteriorly to the larynx. 